\title{
Bidirectional Activity-Dependent Regulation of Neuronal Ion Channel Phosphorylation
}

\author{
Hiroaki Misonou, Milena Menegola, Durga P. Mohapatra, Lauren K. Guy, Kang-Sik Park, and James S. Trimmer \\ Department of Pharmacology, School of Medicine, University of California, Davis, California 95616
}

Activity-dependent dephosphorylation of neuronal Kv2.1 channels yields hyperpolarizing shifts in their voltage-dependent activation and homoeostatic suppression of neuronal excitability. We recently identified 16 phosphorylation sites that modulate Kv2.1 function. Here, we show that in mammalian neurons, compared with other regulated sites, such as serine (S)563, phosphorylation at $S 603$ is supersensitive to calcineurin-mediated dephosphorylation in response to kainate-induced seizures in vivo, and brief glutamate stimulation of cultured hippocampal neurons. In vitro calcineurin digestion shows that supersensitivity of S603 dephosphorylation is an inherent property of Kv2.1. Conversely, suppression of neuronal activity by anesthetic in vivo causes hyperphosphorylation at S603 but not S563. Distinct regulation of individual phosphorylation sites allows for graded and bidirectional homeostatic regulation of Kv2.1 function. $S 603$ phosphorylation represents a sensitive bidirectional biosensor of neuronal activity.

Key words: Kv2.1; potassium channel; calcineurin; hippocampus; intracellular signaling; calcium

\section{Introduction}

Voltage-dependent $\mathrm{K}^{+}(\mathrm{Kv})$ channels are potent suppressors of neuronal excitability and represent attractive candidates for therapeutic modulation of neuronal activity, including neuronal hyperexcitability that occurs in stroke and epileptic patients. Delayed rectifier $\mathrm{Kv}$ currents $\left(I_{\mathrm{K}}\right)$ are important in regulating somatodendritic excitability in hippocampal and cortical pyramidal neurons (Bekkers, 2000; Du et al., 2000; Korngreen and Sakmann, 2000). In these neurons, Kv2.1 is the major component of somatodendritic $I_{\mathrm{K}}$ (Martina and Jonas, 1997; Murakoshi and Trimmer, 1999; Du et al., 2000; Malin and Nerbonne, 2000; Pal et al., 2003). Interestingly, many studies of somatodendritic $I_{\mathrm{K}}$, and of Kv2.1, in pyramidal neurons suggest a function in regulating excitability and $\mathrm{Ca}^{2+}$ influx during periods of repetitive highfrequency firing (Bekkers, 2000; Du et al., 2000; Kang et al., 2000; Korngreen and Sakmann, 2000; Colbert and Pan, 2002). Kv2.1 is highly phosphorylated in resting neurons, but increased neuronal activity induced by seizures and hypoxia in vivo, or glutamate stimulation and induced experimental ischemia in cultured neurons, leads to bulk dephosphorylation of Kv2.1 through a $\mathrm{Ca}^{2+}$ / calcineruin-dependent mechanism (Misonou et al., 2004, 2005). This results in increased amplitude of $I_{\mathrm{K}} / \mathrm{Kv} 2.1$ currents caused by large $(\approx 30 \mathrm{mV})$ hyperpolarizing shifts in voltage-dependent

Received Sept. 12, 2006; revised 0ct. 26, 2006; accepted Nov. 21, 2006.

This work was supported by grants from National Institutes of Health-National Institute of Neurological Disorders and Stroke (NS42225 to J.S.T.) and the American Heart Association (H.M.). D.P.M. and M.M. acknowledge postdoctoral fellowship from the Epilepsy Foundation through the generous support of the American Epilepsy Society and the Milken Family Foundation. We thank Dr. Jeanne Nerbonne for kindly providing the samples from Kv2.1-deficient mice and Drs. JoAnne Engebrecht and Helene Vacher for critical reading of and comments on this manuscript.

Correspondence should be addressed to Dr. James S. Trimmer, Department of Pharmacology, School of Medicine, Room 3502 Genome and Biomedical Sciences Facility, University of California, One Shields Avenue, Davis, CA 95616. E-mail: jtrimmer@ucdavis.edu.

H. Misonou's present address: Department of Biomedical Science, University of Maryland, Baltimore, MD 21201. D01:10.1523/JNEUROSCI.3970-06.2006

Copyright $\odot 2006$ Society for Neuroscience $\quad 0270-6474 / 06 / 2613505-10 \$ 15.00 / 0$ activation (Misonou et al., 2004). The enhanced activity of Kv2.1 provides homeostatic suppression of neuronal activity (Surmeier and Foehring, 2004; Misonou et al., 2005).

Ion channel phosphorylation is a common mechanism to achieve dynamic and reversible modification of localization and function. Ionotropic AMPA-type glutamate receptor subunits are prime examples, whereby surface expression (Ehlers, 2000) and localization and synaptic clustering (Chung et al., 2000) are regulated by reversible phosphorylation. Voltage-gated ion channels are also extensively regulated by phosphorylation (Levitan, 2006). For example, protein phosphatase 1 (PP1)/PP2A-mediated dephosphorylation of a single protein kinase $\mathrm{C}$ (PKC) phosphorylation site on the $\mathrm{Kv} 3.1 \mathrm{~b}$ channel in mammalian auditory neurons in response to auditory stimulation enhances Kv3.1b current and allows for highfrequency firing (Song et al., 2005).

Recently, we found that like endogenous Kv2.1 in neurons, recombinant Kv2.1 in human embryonic kidney HEK293 cells is extensively phosphorylated, and is dephosphorylated after calcineurin activation in response to $\mathrm{Ca}^{2+}$ ionophore or muscarinic stimulation (Mohapatra and Trimmer, 2006). Mass spectrometric-based analyses of Kv2.1 immunopurified from HEK cells identified 16 phosphorylation sites in control cells, 7 of which are subjected to calcineurin-dependent dephosphorylation (Park et al., 2006). Mutation of individual calcineurinsensitive sites led to incremental $(\approx 5-15 \mathrm{mV})$ changes in gating that together contribute to the larger $(\approx 35 \mathrm{mV})$ changes seen after complete dephosphorylation. We generated phosphospecific antibodies against four of the identified phosphorylation sites, at serine (S)453, S563, S603, and S715, and found that these sites are all phosphorylated in rat brain in vivo (Park et al., 2006). Here, we show that two of these sites (S563 and S603) exhibit distinct patterns of activity-dependent regulation in brain neurons in vivo and in cultured hippocampal neurons that underlie graded changes in Kv2.1 function and allow for sensitive, bidirectional homeostatic regulation of neuronal excitability. 


\section{Materials and Methods}

Materials. Kv2.1-deficient mouse brains were generous gift of Dr. Jeanne Nerbonne (Washington University School of Medicine, St. Louis, MO). All other materials were purchased from Sigma (St. Louis, MO) or EMD Biosciences (San Diego, CA) unless noted. The phosphoindependent rabbit KC (Trimmer, 1991) and mouse monoclonal K89/34 (Antonucci et al., 2001) antibodies were raised against a C-terminal peptide 837-853 of Kv2.1. K89/34 can be obtained from NeuroMab (www.neuromab. org), a not-for-profit supplier of monoclonal antibodies administered through the University of California.

Production of phosphospecific antibodies. Antibodies specific for phosphorylated epitopes on Kv2.1 were raised in rabbits against the following phosphorylated peptides: S563P, CEELEMSSMPpSPVAPLPARTEG; S603P, CEATRFSHpSPLASLSSKAGSST, as reported previously (Park et al., 2006). The peptides were conjugated to keyhole limpet hemocyanin and the resultant complex used to generate polyclonal rabbit antisera (Antibodies, Davis, CA).

Kainate-induced seizure animals. All animal use procedures were in strict accordance with the Guide for the Care and Use of Laboratory Animals described by the National Institutes of Health. Adult rats were injected with $15 \mathrm{mg} / \mathrm{kg}$ kainate dissolved in saline or the vehicle. The progression of seizure after injection was assessed by recording the behavioral seizure stage according to Racine's classification (Racine, 1972). A full behavioral seizure, with loss of postural control, was considered as a class 5 motor seizure. Animals that had the class 5 seizure were killed by decapitation and subjected to biochemical analysis as described previously (Misonou et al., 2004), and as summarized below.

Brain membrane preparation. Animals were killed by rapid decapitation either with or without $\mathrm{CO}_{2}$ (inhalation) or $50 \mathrm{mg} / \mathrm{kg}$ sodium pentobarbital (intraperitoneal injection) anesthesia. Rat brains were dissected and used whole or further dissected to obtain hippocampi. Rat brain membrane fractions (RBM) were prepared from either whole brains or hippocampi and treated with purified phosphatases as described previously (Misonou et al., 2006).

Immunoprecipitation of $\mathrm{Kv} 2.1$. RBM (1 mg) was solubilized in $1 \mathrm{ml}$ of lysis buffer containing $1 \%$ Triton X-100, $150 \mathrm{~mm} \mathrm{NaCl}, 1 \mathrm{~mm} \mathrm{NaF}, 1 \mathrm{~mm}$ PMSF, protease inhibitor mixture, and $20 \mathrm{~mm}$ Tris- $\mathrm{HCl}, \mathrm{pH}$ 8.0, for 30 min on ice. Insoluble material was removed by centrifugation at $16,500 \times g$ for $5 \mathrm{~min}$ at $4^{\circ} \mathrm{C}$. The resultant lysate was incubated overnight at $4^{\circ} \mathrm{C}$ with mouse anti-Kv2.1 monoclonal antibody D4/11 (Rhodes et al., 1995) conjugated to agarose beads, or with KC, S453P, S563P, S603P, or $\mathrm{S} 715 \mathrm{P}$ antibodies followed by incubation with protein $\mathrm{G}$ beads. After extensive washes, bound proteins were eluted by boiling the beads in SDS sample buffer for $3 \mathrm{~min}$.

Biochemical analysis of neuronal proteins. Dissociated cultures of embryonic rat hippocampal neurons were prepared as described previously (Misonou et al., 2004; Misonou and Trimmer, 2005). Briefly, neurons were washed twice with HBSS and incubated with drugs as indicated in the figures. The cells were then washed twice with ice-cold Locke's solution (in mM: $154 \mathrm{NaCl}, 5.6 \mathrm{KCl}, 2.3 \mathrm{CaCl}_{2}, 1 \mathrm{MgCl}_{2}, 5$ glucose, 5 HEPES, $\mathrm{pH} 7.4$ ), harvested, and centrifuged at $12,000 \times \mathrm{g}$ for $30 \mathrm{~min}$ at $4^{\circ} \mathrm{C}$. The pellets were extracted by adding SDS sample buffer (2\% SDS, $1 \mathrm{~mm}$ EDTA, $\quad 10 \%$ glycerol, $0.001 \%$ bromophenol blue, $5 \%$ $\beta$-mercaptoethanol, $62.5 \mathrm{~mm}$ Tris- $\mathrm{HCl}, \mathrm{pH} 6.8$ ) and sonicating briefly.

Immunoblots. Proteins were separated by $7.5 \%$ SDS-PAGE and transferred to nitrocellulose membrane. Blots were probed with $\mathrm{KC}$ or K89/ 34 , stripped in stripping buffer ( $2 \%$ SDS, $100 \mathrm{~mm} \beta$-mercaptoethanol, and $62.5 \mathrm{~mm}$ Tris-HCl, $\mathrm{pH} 6.8$ ), and reprobed with S563P antibody, followed by stripping and reprobing with S603P antibody; experiments performed using antibodies in all different orders yielded identical results. Antisera were used at 1:1000-1:1500 dilutions. For peptide absorption experiments, antisera were incubated at $4^{\circ} \mathrm{C}$ overnight with $5 \mu \mathrm{g} / \mathrm{ml}$ phosphorylated or nonphosphorylated peptides conjugated to bovine serum albumin, before being used for immunoblotting. In all cases, blots were incubated with HRP-conjugated secondary antibodies followed by enhanced chemiluminescence reagent (PerkinElmer, Wellesley, MA). Immunoreactive bands were visualized by exposing the blot to $\mathrm{x}$-ray films. Immunoreactive bands were quantified after densitometric scan- ning using NIH Image software (ImageJ) with the profile function. All quantification was performed on at least three different samples.

Immunohistochemistry. Animals were anesthetized with pentobarbital and either perfused immediately or subjected to $\mathrm{CO}_{2}$ inhalation followed by perfusion with $4 \%$ paraformaldehyde. Sagittal brain sections (each 40 $\mu \mathrm{m}$ thick) were prepared from perfusion-fixed animals (Rhodes et al., 2004). Free-floating sections were blocked and permeabilized with $10 \%$ goat serum, $0.3 \%$ Triton X-100 in $0.1 \mathrm{~m}$ phosphate buffer, and incubated overnight in primary antibody vehicle containing S563P or S603P together with $\mathrm{K} 89 / 34$. The sections were then incubated in species-specific Alexa dye-conjugated secondary antibodies (Invitrogen, San Diego, CA) for $1 \mathrm{~h}$. For cultured neurons, cells were fixed with $4 \%$ paraformaldehyde, permeabilized in $0.1 \%$ Triton X-100, blocked in $4 \%$ solution of nonfat dry milk, and stained with S563P or S603P together with K89/34, and Alexa-conjugated secondary antibodies. Fluorescence images were taken using the same exposure time for each set of experiments to compare the signal intensity with a CCD camera installed on an Axiovert $200 \mathrm{M}$ microscope with $63 \times, 1.3$ numerical aperture (NA) lens or $20 \times$, 0.8 NA lens, and an ApoTome coupled to Axiovision software (Zeiss, Oberkochen, Germany).

Electrophysiology. $I_{\mathrm{K}}$ current recordings were made with the whole-cell voltage-clamp configuration as described previously (Misonou et al., 2004). Patch pipettes were pulled from borosilicate glass tubing, heatpolished at the tip to give a resistance of $1-2 \mathrm{M} \Omega$ when filled with the pipette solution (in mM: $140 \mathrm{KCl}, 5 \mathrm{EGTA}, 1 \mathrm{CaCl}_{2}, 2 \mathrm{MgCl}_{2}, 10$ glucose, 10 HEPES, pH 7.3). Currents were recorded at a $10 \mathrm{kHz}$ sampling rate and filtered at $2 \mathrm{kHz}$ by a digital Bessel filter. All currents were capacitance-, series resistance-compensated and leak-subtracted by standard $P / n$ procedure. External solution contained $0.1 \mu \mathrm{M}$ tetrodotoxin, $140 \mathrm{~mm} \mathrm{NaCl}, 5 \mathrm{~mm} \mathrm{KCl}, 2 \mathrm{~mm} \mathrm{CaCl}$, 2 mм MgCl 2,10 mм glucose, 10 mM HEPES, pH 7.3. Glutamate $(10 \mu \mathrm{M})$ was applied to neurons for 10 min using a perfusion system. For the recovery experiments, currents were recorded from neurons incubated for $2 \mathrm{~h}$ in external solution in the absence or presence of $1 \mu \mathrm{M}$ staurosporine after an initial $10 \mathrm{~min}$ glutamate stimulation. All recordings were performed at room temperature $\left(23-25^{\circ} \mathrm{C}\right)$. The membrane potential was held at -80 or $-100 \mathrm{mV}$ and depolarized from the holding potential to voltages up to $+80 \mathrm{mV}$ in 10 $\mathrm{mV}$ increments for $200 \mathrm{~ms}$ with an interpulse interval of $1.2 \mathrm{~s}$. Before the start of the test depolarization, a single prepulse to $-10 \mathrm{mV}$ was given for $30 \mathrm{~ms}$ to inactivate majority of the transient outward $\mathrm{K}^{+}$currents. Peak outward sustained $\mathrm{K}^{+}$currents $(I)$ were measured $100 \mathrm{~ms}$ into the pulse at each depolarizing potential, and were converted to conductance $(G)$ using the following equation: $G=I /\left(V-E_{\mathrm{K}}\right)$. The Nernst $\mathrm{K}^{+}$equilibrium potential $\mathrm{E}_{\mathrm{K}}$ was calculated as $-84 \mathrm{mV}$. The normalized conductance was plotted against the test potential $(V)$ and fitted to a single Boltzmann equation $G=G_{\max } /\left(1+\exp \left[-\left(V-G_{1 / 2}\right) / k\right]\right)$, where $G_{\max }$ is the maximum conductance, $G_{1 / 2}$ is the test potential at which the $I_{\mathrm{K}}$ channels have a half-maximal conductance, and $k$ represents the slope of the activation curve.

In vitro phosphorylation and dephosphorylation of Kv2.1. Kv2.1 solubilized in 1\% Triton X-100 lysis buffer was purified from RBM (1 mg of protein) by immunoprecipitation using D4/11 beads $(50 \mu \mathrm{l})$ as described above. For in vitro dephosphorylation, $10 \mu \mathrm{l}$ of beads were resuspended in $100 \mu \mathrm{l}$ of calcineurin reaction buffer $(100 \mathrm{~mm} \mathrm{NaCl}, 6 \mathrm{mM} \mathrm{MgCl} 2,2$ $\mathrm{mm} \mathrm{CaCl}_{2}, 1 \mathrm{~mm}$ dithiothreitol, $0.1 \mathrm{mg} / \mathrm{ml}$ bovine serum albumin, $2.5 \mu \mathrm{M}$ purified bovine brain calmodulin, protease inhibitor mixture, $25 \mathrm{U}$ of recombinant human calcineurin, and $20 \mathrm{~mm}$ Tris- $\mathrm{HCl}, \mathrm{pH}$ 7.5), or alkaline phosphatase reaction buffer $(0.1 \%$ SDS, $0.1 \mathrm{~mm}$ EDTA, and $50 \mathrm{~mm}$ Tris-HCl, $\mathrm{pH} 8.5)$. NaF (1 mM) was added to the control reaction. The reaction mixture was incubated for $2 \mathrm{~h}$ at $32^{\circ} \mathrm{C}$, and the reaction was terminated by boiling in SDS sample buffer. For in vitro phosphorylation, the beads were resuspended in $100 \mu \mathrm{l}$ of protein kinase $\mathrm{C}$ reaction buffer ( $5 \mathrm{~mm} \mathrm{MgCl}_{2}, 1 \mathrm{~mm}$ dithiothreitol, $2 \mathrm{~mm} \mathrm{CaCl}, 100 \mu \mathrm{M} \mathrm{ATP}, 1 \times$ PKC micelle, $2.5 \mathrm{ng}$ of purified rat brain protein kinase $\mathrm{C}$, and $20 \mathrm{~mm}$ Tris$\mathrm{HCl}, \mathrm{pH} 7.5)$, and incubated for $1 \mathrm{~h}$ at $32^{\circ} \mathrm{C}$. PKC micelle $(10 \times)$ was prepared by dissolving $470 \mu \mathrm{g}$ of dried $\mathrm{L}-\alpha$-phosphatidylserine in $10 \mu \mathrm{l}$ of $10 \%$ Triton X-100, $40 \mu \mathrm{l}$ of $20 \mathrm{~mm}$ Tris- $\mathrm{HCl}, \mathrm{pH} 7.5$, and $0.8 \mu \mathrm{l}$ of $1 \mathrm{~mm}$ phorbol 12-myristate 13-acetate (Sipeki et al., 2002). After washing with 

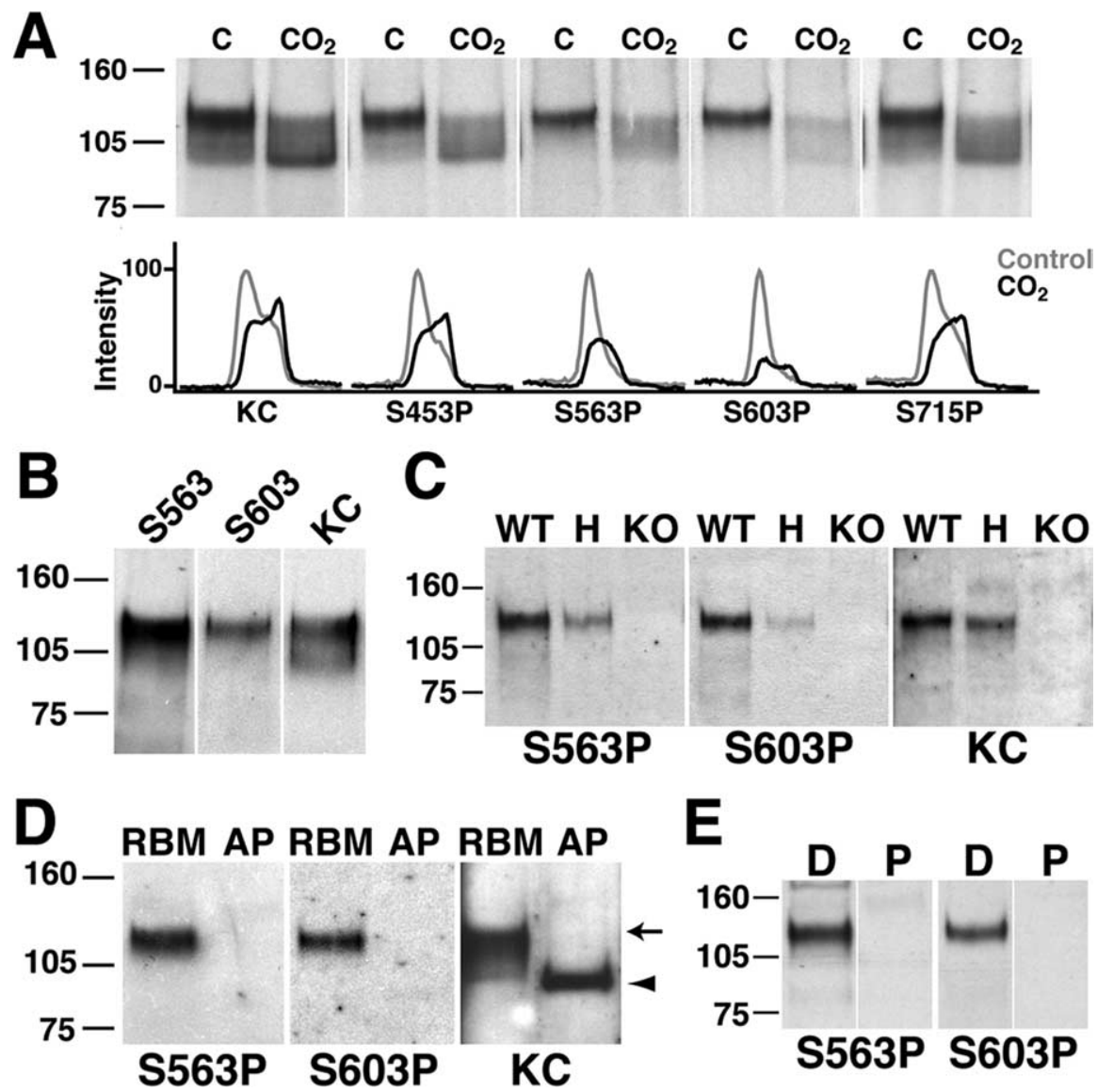

Figure 1. Characterization of the S563P and S603P phosphospecific anti-Kv2.1 antibodies. $\boldsymbol{A}$, Analysis of anti-Kv2.1 phosphospecific antibodies against Kv2.1 dephosphorylation in response to brain ischemia. RBM fractions were prepared from rats treated without $(\mathrm{C})$ or with $\mathrm{CO}_{2}$ gas. Proteins were extracted in lysis buffer, subjected to immunoprecipitation with the indicated antibodies, size fractionated by SDS-PAGE, and analyzed for Kv2.1 by immunoblotting with K89/34. Numbers to the left indicate mobility of molecular weight standards in kilodaltons. The bottom panel shows the respective line scan analysis of each immunoblot. $\boldsymbol{B}, \mathrm{Kv} 2.1$ was immunopurified from detergent extracts of RBM with mouse monoclonal antibody D4/11, size fractionated by SDS-PAGE, immunoblotted, and probed with S563P, S603P, or KC antibodies. C, Immunoblot of mouse brain membrane fractions from wild-type and Kv2.1-deficient mice with S563P, S603P, or KC antibodies. WT, Wild type; H, heterozygous knockout; K0, homozygous knock-out. D, RBM were incubated without (RBM) or with (AP) alkaline phosphatase for $2 \mathrm{~h}$ at $37^{\circ} \mathrm{C}$. Proteins (20 $\mu \mathrm{g} /$ lane) were extracted in SDS sample buffer, size fractionated by SDS-PAGE, and analyzed for total Kv2.1 with S563P, S603P, or KC antibodies. The arrow indicates the fully phosphorylated $\left(M_{\mathrm{r}} \approx 120 \mathrm{kDa}\right)$ form of Kv2.1, and the arrowhead points to the fully dephosphorylated form. $\boldsymbol{E}$, Peptide adsorption of the phosphospecific antibodies. Blots of RBM ( $20 \mu \mathrm{g}$ protein/lane) were probed with the phosphospecific antibodies after overnight incubation with their respective phosphorylated (P) or dephosphorylated (D) peptides.

(Misonou et al., 2005), we found that the S563 and S603 phosphorylation sites are more sensitive to ischemia-induced dephosphorylation than are S453 and S715 (Fig. 1A). The phosphoindependent rabbit polyclonal antibody KC (Trimmer, 1991) was used to measure the total level of Kv2.1 protein in these samples and to verify that changes in phosphospecific antibody immunoreactivity were not attributable to changes in Kv2.1 protein levels but in phosphorylation state.

Before pursuing additional studies of in vivo regulation of phosphorylation at $\mathrm{S} 563$ and S603, we further validated the S563P and $\mathrm{S603P}$ antibodies. To verify that these antibodies specifically recognize Kv2.1, we used a phosphoindependent anti-Kv2.1 antibody to immunopurify Kv2.1 from brain, and found that immunoreactivity for S563P and S603P was enriched in parallel with that for $\mathrm{KC}$, showing that these antibodies share the same molecular target (Fig. 1B). Moreover, immunoreactivity for S563P and S603P (and KC) that was present in samples prepared from the brains of wild-type mice was completely eliminated in Kv2.1-deficient mice, and appropriately diminished in heterozygotes (Fig. 1C). We further verified the phosphospecificity of S563P and S603P on immunoblots by analyzing control and alkaline phosphatase (AP)-treated RBM with $\mathrm{KC}$, or with S563P and S603P. As shown in Figure $1 D$, KC detected highly phosphorylated $\left(M_{\mathrm{r}} \approx 120 \mathrm{kDa}\right.$; arrow $)$ and dephosphorylated $\left(M_{\mathrm{r}} \approx 100 \mathrm{kDa}\right.$; arrowhead $)$ forms of Kv2.1, as well as multiple Kv2.1 species between 100 and $120 \mathrm{kDa}$ in the control RBM samples that presumably represent Kv2.1 with different levels of in vivo phosphorylation. In contrast to $\mathrm{KC}$, S563P and S603P preferentially detected the highly phosphorylated $\left(M_{\mathrm{r}} \approx 120\right.$ $\mathrm{kDa}$ ) form of Kv2.1, and exhibited no immunoreactivity against Kv2.1 with inter-

ice-cold lysis buffer, bound protein was eluted by adding SDS sample buffer and boiling for $3 \mathrm{~min}$, and subjected to immunoblotting.

Statistical analyses. All data are presented as the mean \pm SEM. Normal data were compared by a two-sided $t$ test. A $t$ value of $p<0.05$ was considered to be statistically significant. Regression analyses were done with GraphPad Prism software (San Diego, CA).

\section{Results}

Characterization of phosphospecific S563 and

S603 antibodies

We recently generated four rabbit polyclonal phosphospecific antibodies, S453P, S563P, S603P, and S715P, that selectively recognize Kv2.1 phosphorylated at these sites that are located in the Kv2.1 cytoplasmic C terminus (Park et al., 2006). Serine-toalanine mutations at each of these sites yields significant (9-16 $\mathrm{mV}$ ) changes in the voltage-dependent activation of recombinant Kv2.1 (Park et al., 2006). When assayed on RBM samples from animals subjected to ischemia induced in vivo by $\mathrm{CO}_{2}$ inhalation mediate levels of phosphorylation in the control RBM sample, or the completely dephosphorylated form Kv2.1 in the AP-treated RBM sample (Fig. 1D). Finally, phosphospecificity of S563P and $\mathrm{S} 603 \mathrm{P}$ was validated in peptide competition experiments using the respective phosphorylated and nonphosphorylated peptides. As shown in Figure $1 E$, preincubation with phosphorylated but not nonphosphorylated cognate peptides completely eliminated the immunoreactivity of both S563P and S603P against RBM. We also verified the linearity of immunoblot signals obtained using the phosphospecific antibodies by analyzing different amounts of RBM and by measuring the optical density of the obtained signals (supplemental Fig. 1A, $B$, available at www.jneurosci.org as supplemental material). The results together establish the validity of using these phosphospecific antibodies in accurately measuring the level of Kv2.1 phosphorylated at these sites in mammalian brain preparations.

The S563 and S603 phosphospecific antibodies also specifi- 
cally stained Kv2.1 clusters in brain sections from adult rats that had been doublestained with a phosphoindependent mouse monoclonal anti-Kv2.1 antibody. This was especially apparent in sections of neocortical pyramidal (Fig. 2A) and subicular (Fig. $2 B$ ) neurons. Although S563P and $\mathrm{S} 603 \mathrm{P}$ stained most neurons in these regions, certain neurons (for example, layer $\mathrm{V}$ cortical pyramidal neurons) had a more intense signal than others (note the relative intensity of yellow vs green staining in Fig. 2A), indicating that in adult brain the extent of phosphorylation at these sites may differ in different neurons. In a single neuron, individual clusters also exhibited heterogeneity in the levels of S563P and S603P staining (Fig. 2B; supplemental Fig. 2, available at www. jneurosci.org as supplemental material). Brain ischemia as induced by $\mathrm{CO}_{2}$ inhalation, and which results in dephosphorylation of Kv2.1 (Misonou et al., 2005) (Fig. $1 A$; supplemental Fig. $1 A$, available at www.jneurosci.org as supplemental material) and dispersion of Kv2.1 clusters (Misonou et al., 2005), also yields extensive loss of immunoreactivity for both phosphospecific antibodies (Fig. 3). These results together demonstrate the phosphospecificity of the S563P and S603P antibodies.

\section{Differential regulation of Kv2.1 \\ phosphorylation at S563 and S603 during brain development}

Kv2.1 exhibits changes in $M_{\mathrm{r}}$ during brain development as a result of changes in phosphorylation state (Trimmer, 1993) (H. Misonou and J. S. Trimmer, unpublished data). To obtain additional insights into regulation of Kv2.1 phosphorylation in vivo, we investigated the phosphoryla-

tion state at S563 and S603 during development. We performed immunoblots with sequential reprobing using KC, S563P and S603P (for details, see Materials and Methods) to reliably compare the levels of phosphorylation at individual sites in RBM samples prepared from brains of rats from postnatal day 2 (P2) through adulthood (Fig. 4A). Unlike Kv1 channels, such as Kv1.2 (Fig. 4A), which exhibit a relatively late postnatal expression (Maletic-Savatic et al., 1995), the expression level of total brain Kv2.1 is relatively constant throughout postnatal development as shown on the KC immunoblot (Fig. 4A) (Trimmer, 1993). The level of S603 phosphorylation approximately parallels the levels of total Kv2.1 at each developmental time point (Fig. 4A). However, phosphorylation at S563 was reduced in the brains of neonatal animals, with relatively low levels present at P2 and P5, and exhibited a sharp increase during the second postnatal week to reach adult levels by $\mathrm{P} 14$. The distinct patterns of phosphorylation at these two sites may reflect requirements for different functional states of Kv2.1 during different periods of rat brain development.
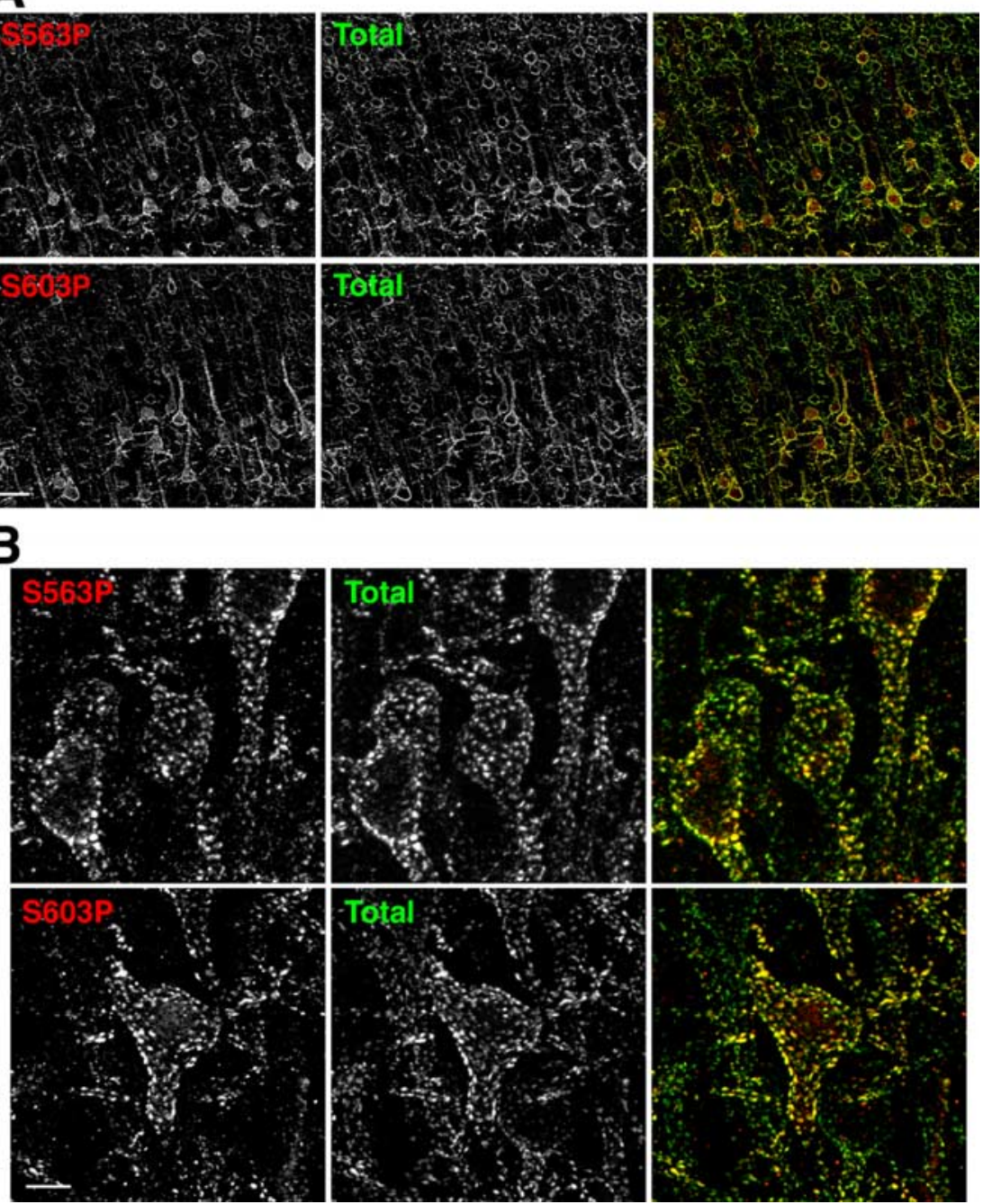

Figure 2. Immunostaining of rat brain with phosphospecific antibodies. Rat brain sections were double immunofluorescence neurons in the region. Scale bar, $50 \mu \mathrm{m}$. B, Pyramidal neurons in the subiculum. Overlaid images show the heterogeneity of $\$ 563$ and $\mathbf{S 6 0 3}$ phosphorylation in Kv2.1 clusters. Scale bar, $10 \mu \mathrm{m}$.

\section{Dephosphorylation of S563 and S603 in kainate-induced seizure brains}

We next addressed how increased neuronal activity in adult brain regulates in vivo Kv2.1 phosphorylation at S563 and S603. Hippocampi from rats subjected to the kainate model of acute seizures, in which Kv2.1 is significantly dephosphorylated in response to increased neuronal activity (Misonou et al., 2004), were analyzed by immunoblot. KC immunoblots revealed that Kv2.1 in these samples was in fact significantly dephosphorylated in seizure hippocampi, as revealed by a prominent shift in $M_{\mathrm{r}}$ relative to those from hippocampi of control animals (Fig. $4 B$ ). The level of $M_{\mathrm{r}} \approx 120 \mathrm{kDa}$ phosphorylated Kv2.1 in the samples from kainate-seized rats was significantly reduced (by $\approx 35 \%$ ) compared with control samples. Immunoreactivity for both S563P and S603P was also significantly decreased in the samples from kainate-seized rats, whereas the overall level of Kv2.1 on the KC immunoblots was not (as determined by measurement of the entire microheterogeneous immunoreactive population), demonstrating activity-dependent dephosphorylation at these sites in vivo (Fig. $4 B, C$ ). However, the extent of dephosphorylation at 

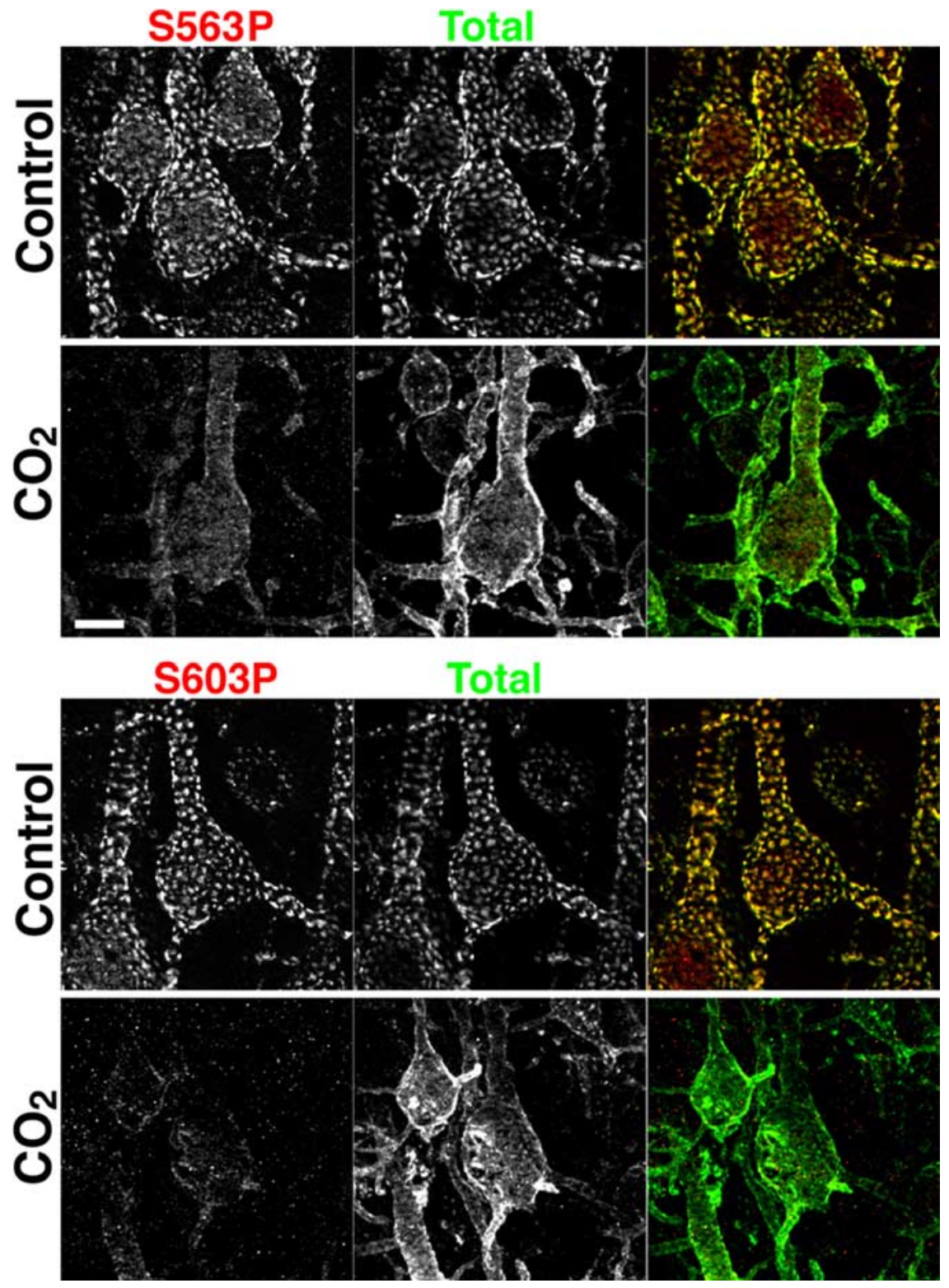

Figure 3. Dephosphorylation of $\$ 563$ and $\$ 603$, and dispersion of Kv2.1 clusters, by brain ischemia. Rat brain sections prepared from control and $\mathrm{CO}_{2}$-treated rats were double immunofluorescence stained with $5563 \mathrm{P}$ or $5603 \mathrm{P}$ (red) together with $\mathrm{K} 89 / 34$ (Total; green). Images were taken by a fluorescence microscope equipped with ApoTome from the cerebral cortex. Scale bar, $10 \mu \mathrm{m}$.

S603 phosphorylation was more evident than for that at S563, as revealed by line scan analyses of immunofluorescence signals (Fig. 5B).

To further address whether differential dephosphorylation at S563 and S603 occurs in response to changes in neuronal activity, neurons were treated with $10 \mu \mathrm{M}$ glutamate for different times and Kv2.1 phosphorylation analyzed by quantitative immunoblot. As previously reported (Misonou et al., 2004), and as shown in Figure $6 \mathrm{~A}$, glutamate stimulation induced graded reductions in the $M_{\mathrm{r}}$ of $\mathrm{Kv} 2.1$ on $\mathrm{KC}$ immunoblots, but no change in the overall level of Kv2.1 protein. Phosphorylation of S563 exhibited a marked decrease with a time course similar to the decrease in the levels of the major $M_{\mathrm{r}} \approx 120 \mathrm{kDa}$ phosphorylated form of Kv2.1 on the KC immunoblot (Fig. 6A). However, phosphorylation at $\mathrm{S} 603$ was much more sensitive to glutamate stimulation such that the S603P immunoblot signal virtually disappeared within $1 \mathrm{~min}$ of glutamate stimulation (Fig. 6A), a time point when there was little discernible change in the $M_{\mathrm{r}}$ of Kv2.1 on the KC blots. Dephosphorylation at both S563 and S603 was calcineurin dependent, such that the effect of glutamate was blocked by a calcineurin inhibitor cyclosporin A (data not shown) as previously reported for changes in $\mathrm{Kv} 2.1 M_{\mathrm{r}}$ (Misonou et al., 2004).

Quantitative analyses of these data (Fig. $6 \mathrm{~B}$ ) revealed that the time course of S563 dephosphorylation in response to $10 \mu \mathrm{M}$ glutamate stimulation was fit by an exponential decay curve $\left(r^{2}=0.87\right)$ with a response half-time $\left(t_{1 / 2}\right)$ of $5.6 \mathrm{~min}$, which is statistically indistinguishable $(p=0.77)$ from the time course of loss of the $M_{\mathrm{r}} \approx$ $120 \mathrm{kDa}$ band on the $\mathrm{KC}$ immunoblot (Fig. 6B, inset). However, the kinetics of glutamate-induced loss of phosphorylation at $\mathrm{S} 603$ was best fit by a curve with a

these two sites differed significantly, because phosphorylation at S563 was reduced by $\approx 40 \%$ in the samples from kainate-seized rats compared with control values, whereas that at $\mathrm{S} 603$ was reduced by $\approx 85 \%$ (Fig. $4 B, C$ ).

\section{Differential dephosphorylation of S563 and S603 in cultured hippocampal neurons}

Glutamate stimulation of cultured hippocampal neurons results in neuronal hyperexcitability, increased cytoplasmic $\mathrm{Ca}^{2+}$, calcineurin-dependent Kv2.1 dephosphorylation, and lateral dispersion of surface Kv2.1 clusters (Misonou et al., 2004). Immunofluorescence staining of cultured neurons with S563P and S603P (Fig. 5A) revealed that in control neurons most of the Kv2.1 clusters contained channels phosphorylated at these sites. Glutamate stimulation (10 $\mu \mathrm{M} ; 10 \mathrm{~min})$ induced lateral dispersion of Kv2.1 clusters and decreased the levels of S563 and S603 phosphorylation (Fig. 5A). However, the decrease in the level of $t_{1 / 2}=0.4 \mathrm{~min}\left(r^{2}=0.97\right)$. This 14 -fold difference in the rates of dephosphorylation at these two different sites demonstrate a fundamental and significant $(p<0.0001$ by regression analysis) difference in the activity- and calcineurin-dependent regulation of Kv2.1 phosphorylation at S563 and S603, and strongly suggest that dephosphorylation of Kv2.1 is a multiple-step event that yields graded regulation of Kv2.1. It should be noted that, because the loss of $\mathrm{S} 603$ phosphorylation does not affect the electrophoretic mobility of Kv2.1 (Fig. 6A), measurements of the decay of the $M_{\mathrm{r}} \approx 120 \mathrm{kDa}$ band on the KC immunoblot (Fig. 6B, insets) do not reflect the rapid loss of $\mathrm{S} 603$ phosphorylation.

\section{Graded modulation of Kv2.1 function in cultured neurons}

We next examined the temporal relationship between the distinct kinetics of glutamate-induced dephosphorylation of neuronal Kv2.1 at S563 and S603, and the functional modulation of neuronal $I_{\mathrm{K}}$, the bulk of which $(>80 \%)$ comprises Kv2.1 channels 
A
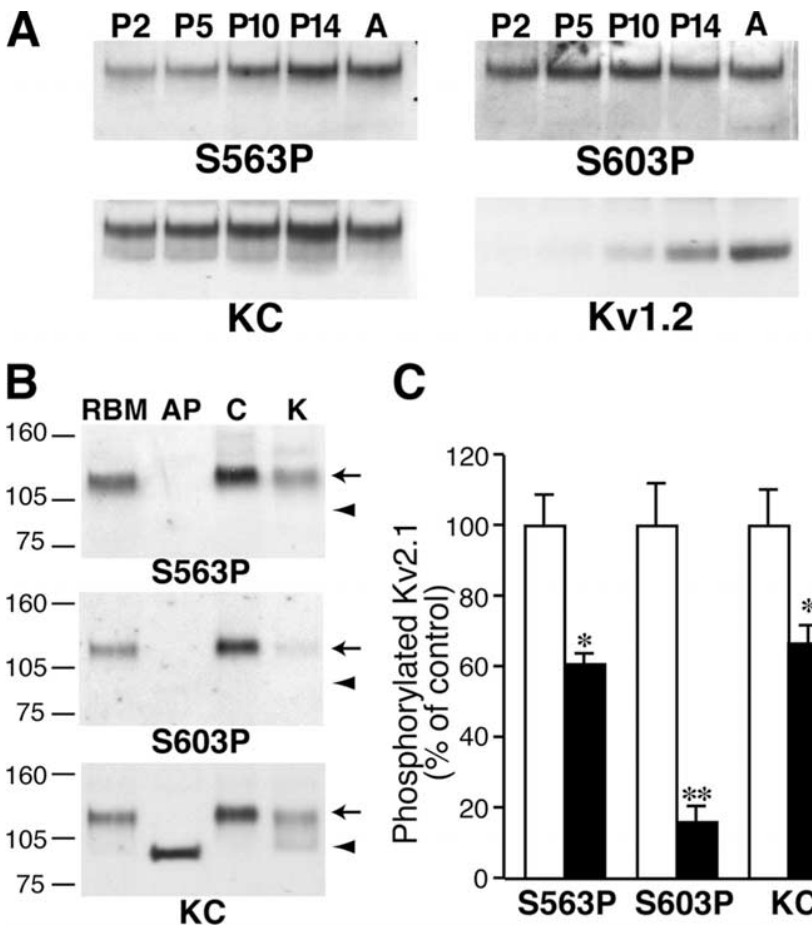

C

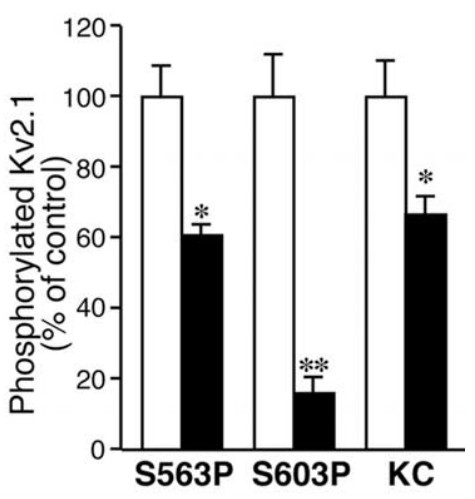

Figure 4. Differential regulation of brain Kv2.1 phosphorylation at S563 and S603. A, RBM fractions prepared from $\mathrm{P2}$, P5, P10, P14, and adult animals were fractionated on SDS-PAGE and analyzed by immunoblotting with $\mathrm{S563P}, \mathrm{S603P}$, or KC, as indicated. Each lane contains $20 \mu \mathrm{g}$ of protein. $\boldsymbol{B}$, Dephosphorylation of Kv2.1 at $\mathrm{S} 563$ and $\mathrm{S} 603$ in kainate-induced seizure brains. Hippocampal membrane proteins ( $20 \mu \mathrm{g} / \mathrm{lane}$ ) from control (C) and kainate-treated (K) animals were size fractionated by SDS-PAGE, and resultant immunoblots were probed with S563P, S603P, or KC as indicated. RBM, Rat whole brain membrane fraction; AP, RBM digested with alkaline phosphatase. The arrow indicates the position of the fully phosphorylated form of Kv2.1, and the arrowhead points to that of the fully dephosphorylated form. C, Levels of Kv2.1 with phosphorylation at $\$ 563$ and $\mathbf{6 6 0 3}$. Hippocampal membrane fractions from three control (open bars) and three kainate-treated animals (filled bars) were used for the analysis. The level of the major $\left(M_{\mathrm{r}} \approx 120 \mathrm{kDa}\right.$ ) phosphorylated form of Kv2.1 in the KC blot was also quantified. Data are mean $\pm \operatorname{SEM}(n=3) .{ }^{*} p<0.05$ and ${ }^{* *} p<0.005$ versus control.

(Murakoshi and Trimmer, 1999; Misonou et al., 2005). Glutamate $(10 \mu \mathrm{M})$ was locally perfused onto cultured neurons for 0-10 min under whole-cell patch-clamp recording conditions. As shown previously (Murakoshi and Trimmer, 1999) and in Figure $6 C$, before glutamate stimulation, whole-cell $I_{\mathrm{K}} / \mathrm{Kv} 2.1$ conductance exhibited a relatively depolarized (high-threshold) voltage dependence of activation, with a half-maximal activation potential $\left(G_{1 / 2}\right)$ of $16.4 \pm 0.4 \mathrm{mV}$. Graded hyperpolarizing shifts in the voltage dependence of activation were observed in response to glutamate stimulation with a half-maximal effect obtained $\sim 5$ min after stimulation with this dose of glutamate (Fig. $6 C)$. As such, the time course of the overall modulation of Kv2.1based $I_{\mathrm{K}}$ is similar to that of S563 and not S603. However, significant changes $(p=0.005)$ in voltage-dependent activation were observed as early as $2 \mathrm{~min}$ of stimulation (Fig. $6 C$ ), within the time frame of $\mathrm{S} 603$ dephosphorylation. As in heterologous cells dephosphorylation of Kv2.1 at as many as seven sites sum in a nonlinear manner to underlay the large $(\approx 25 \mathrm{mV})$ changes in voltage-dependent activation observed in response to stimulation with $\mathrm{Ca}^{2+}$ ionophore (Park et al., 2006), the distinct temporal patterns of dephosphorylation of Kv2.1 at S563 and S603 may yield the graded changes in Kv2.1-based $I_{\mathrm{K}}$ on glutamate stimulation.
Inherent differences in sensitivity of S563 and S603 phosphorylation sites to in vitro calcineurin digestion We next addressed the mechanistic basis for the observed differences in the sensitivity of Kv2.1 phosphorylation at S563 and S603 to calcineurin-mediated dephosphorylation. Rat brain Kv2.1 was immunopurified, subjected to digestion with purified calcineurin in vitro, and analyzed for phosphorylation at S563 and S603. In vitro calcineurin digestion was less efficient in dephosphorylating Kv2.1 than either calcineurin-dependent dephosphorylation in vivo, or in vitro AP digestion, as evidenced by the limited degree of the shift in the $M_{\mathrm{r}}$ of calcineurin-digested Kv2.1 on SDS gels (Fig. 7A). Under these conditions, whereas S563 was demonstrably dephosphorylated as indicated by a loss of S563P immunoreactivity, a large fraction $(\approx 50 \%)$ of phosphorylation at this site remained even after $2 \mathrm{~h}$ of digestion (Fig. 7A). However, in vitro calcineurin digestion resulted in almost complete loss of S603 phosphorylation (Fig. 7A). That the pattern of S563 and S603 dephosphorylation in response to in vitro calcineurin digestion is so similar to that observed with kainate stimulation in vivo, or short (1-5 min) glutamate stimulation of cultured neurons, suggests that the different sensitivities of these sites is dictated mostly by differences in calcineurin sensitivity inherent in the structure of Kv2.1 and not factors contributed by the cellular environment.

\section{Distinct sensitivity of the two Kv2.1 phosphorylation sites to} poststimulus recovery

The effects of activity-dependent dephosphorylation of Kv2.1 in cultured hippocampal neurons are reversible, with recovery of the $M_{\mathrm{r}} \approx 120 \mathrm{kDa}$ phosphorylated form of Kv2.1, and Kv2.1 clustering and function, within $\approx 2 \mathrm{~h}$ of glutamate washout (Misonou et al., 2004). To obtain insights into whether S563 and S603 are distinctly regulated during recovery, cultured neurons were incubated with glutamate to induce Kv2.1 dephosphorylation, followed by a $2 \mathrm{~h}$ glutamate-free recovery period to allow for restoration of phosphorylated Kv2.1. The recovery of Kv2.1 phosphorylation was evident by shifts in $M_{\mathrm{r}}$ of Kv2.1 on the KC immunoblot (Fig. 7B). Phosphorylation at both S563 and S603 was also substantially recovered after the $2 \mathrm{~h}$ recovery period (Fig. $6 B$ ).

The bulk recovery of Kv2.1 phosphorylation during the washout period was partially suppressed by the presence of $100 \mathrm{nM}$ staurosporine (Fig. 7B), suggesting the involvement of a staurosporine-sensitive kinase. Staurosporine is a broad spectrum protein kinase inhibitor that potently blocks PKC, and also inhibits protein kinases $\mathrm{A}$ and $\mathrm{G}, \mathrm{Ca}^{2+} /$ calmodulin-dependent kinases, and myosin light chain kinases with a lower potency (Meggio et al., 1995). Rephosphorylation of Kv2.1 at S563 and S603 exhibited distinct sensitivities to the staurosporine effects, such that staurosporine completely blocked the recovery of phosphorylation at $S 603$, but only partially inhibited recovery of phosphorylation at S563 (Fig. 7B). To determine whether the differences in staurosporine sensitivity are attributable to inherent differences in sensitivity to PKC-mediated phosphorylation, immunopurified rat brain Kv2.1 that had been completely dephosphorylated by in vitro AP digestion was subjected to in vitro phosphorylation with purified PKC. This resulted in a slight increase in the $M_{\mathrm{r}}$ of $\mathrm{Kv} 2.1$ on the $\mathrm{KC}$ immunoblot, and in the reappearance of S603P but not S563P immunoreactivity (Fig. 7C). These results suggest that, in addition to inherent differences in sensitivity to calcineurin-mediated dephosphorylation, S563 and S603 are also differentially regulated by phosphorylation in vivo and in vitro.

To obtain additional insights into the impact of changes in S603 phosphorylation on Kv2.1 function, we examined the effect of staurosporine on the recovery of functional modulation of 

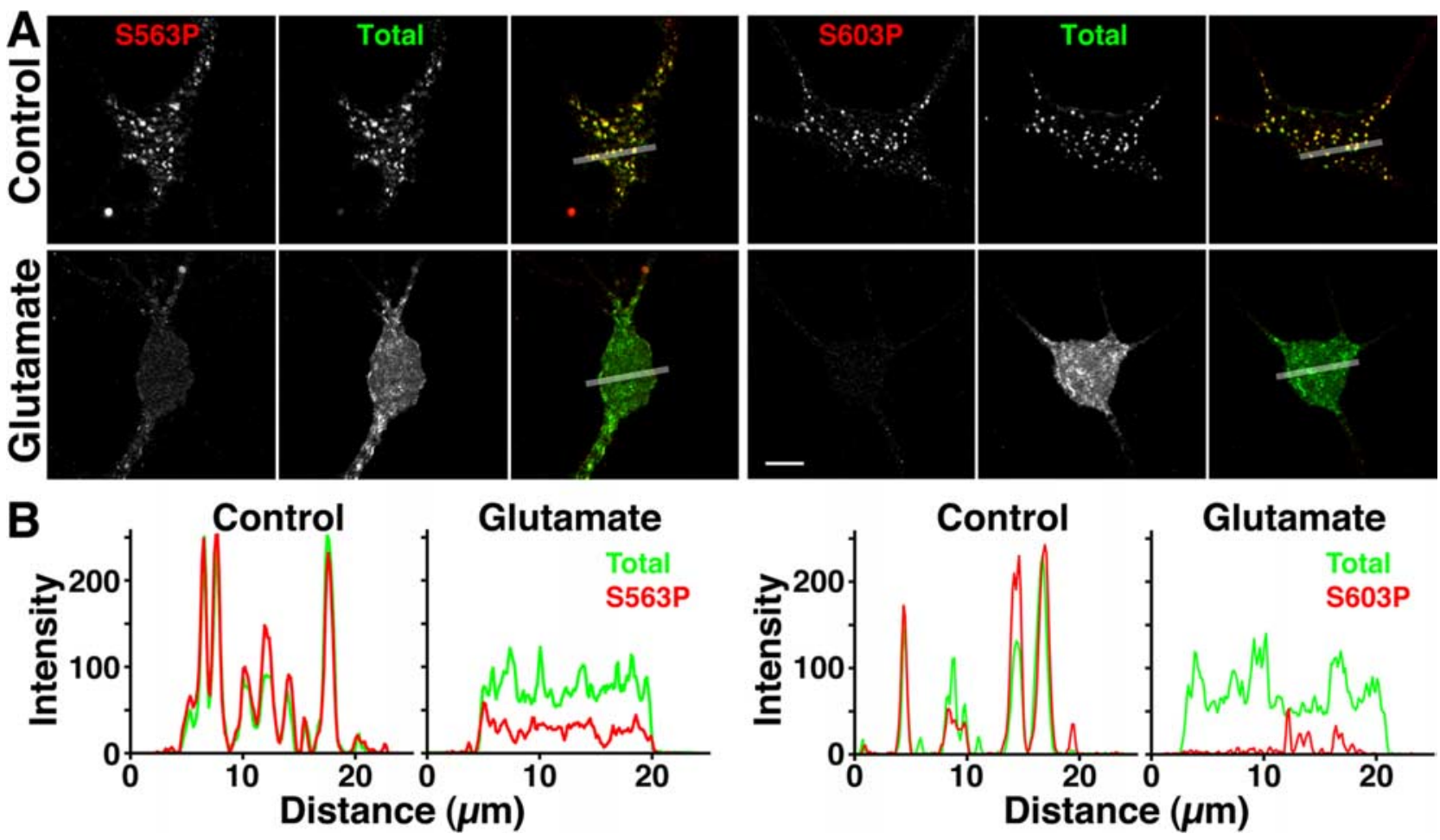

Figure 5. Dephosphorylation of $\$ 563$ and S603, and dispersion of Kv2.1 clusters in cultured hippocampal neurons stimulated with glutamate. $A$, Cultured hippocampal neurons (21 DIV) were incubated without (Control) or with $10 \mu \mathrm{m}$ glutamate (Glutamate) for 10 min, fixed with 4\% paraformaldehyde, permeabilized, and double immunofluorescence stained with S563P or S603P (red) together with K89/34 (Total; green). Scale bar, $10 \mu \mathrm{m} . \boldsymbol{B}$, Line scan analysis of immunofluorescence staining. The intensity of the fluorescence signal was measured over a $25 \mu \mathrm{m}$ segment on the neuronal cell body at the sites indicated by the white lines in $\boldsymbol{A}$. The signal intensity is presented on an arbitrary scale of $0-255$ (black-white) plotted against distance (in micrometers).

$\mathrm{Kv} 2.1 / I_{\mathrm{K}}$ after glutamate stimulation. Neurons were incubated with $10 \mu \mathrm{M}$ glutamate to induce $\mathrm{Kv} 2.1 / I_{\mathrm{K}}$ modulation, followed by a $2 \mathrm{~h}$ recovery period as described above. We previously showed (Misonou et al., 2004) that the glutamate-induced hyperpolarizing shift in the voltage-dependent activation of Kv2.1 recovers within $2 \mathrm{~h}$ of glutamate washout, as shown in Figure $7 D$. In this experiment, the $G_{1 / 2}$ value shifted from the control value of $16.9 \pm 0.3$ to $-9.3 \pm 1.4 \mathrm{mV}(p<0.0001)$ with glutamate treatment. The voltage-dependent activation of $\mathrm{Kv} 2.1 / I_{\mathrm{K}}$ exhibited substantial but not full recovery after $2 \mathrm{~h}\left(G_{1 / 2}=11.6 \pm 0.3 \mathrm{mV}\right)$. However, inclusion of staurosporine during the recovery period completely blocked the recovery of $\mathrm{Kv} 2.1 / I_{\mathrm{K}}$, such that the voltage-dependent activation curve remained essentially unchanged from that in glutamate-treated neurons without recovery $\left(G_{1 / 2}=-7.9 \pm 0.9 \mathrm{mV} ; p=0.42\right.$ when compared with glutamate-treatment alone). These results strongly suggest that staurosporine-sensitive phosphorylation of Kv2.1, including that which occurs at S603, has a significant role in determining voltage-dependent activation of Kv2.1 in neurons.

\section{Hyperphosphorylation of Kv2.1 by suppression of neuronal activity in vivo}

We next addressed whether homeostatic, activity-dependent regulation of Kv2.1 phosphorylation was bidirectional, that is whether increased Kv2.1 phosphorylation occurs in response to suppression of neuronal activity. To suppress neuronal activity in vivo, adult rats were subjected to deep pentobarbital anesthesia. As shown in Figure $8 A, \mathrm{Kv} 2.1$ in the brains of rats anesthetized by intraperitoneal injection of $50 \mathrm{mg} / \mathrm{kg}$ pentobarbital exhibited a slightly increased $M_{\mathrm{r}}$ on the $\mathrm{KC}$ immunoblot, but no change in the overall level of Kv2.1 ( $p>0.05$ ) (data not shown). We also observed a slight and statistically insignificant $(p>0.05)$ in- crease in phosphorylation of S563 in these samples (Fig. 8A,B). In contrast, phosphorylation of $\mathrm{S} 603$ was dramatically and significantly increased $(\approx 3.4$-fold; $p=0.0073)$ in the brains of pentobarbital-anesthetized rats (Fig. $8 A, B$ ). These results indicate that spontaneous activity of brain neurons maintains steadystate phosphorylation of S603 at an intermediate level, allowing for bidirectional regulation after altered neuronal activity.

\section{Discussion}

Distinct patterns of regulation of Kv2.1 phosphorylation Kv2.1 is heavily phosphorylated, with at least 16 sites covalently modified with phosphate in intact cells (Park et al., 2006). Phosphorylation is the major posttranslational modification on Kv2.1 (Shi et al., 1994; Shi and Trimmer, 1999). Extensive phosphorylation of Kv2.1 affords an opportunity for large, reversible changes in protein function. Dephosphorylation of Kv2.1 yields $\approx 30 \mathrm{mV}$ shifts in its voltage-dependent gating (Misonou et al., 2004), which can profoundly affect activity of Kv2.1 channels and neuronal firing. Most other extensively phosphorylated proteins exhibit threshold or switch-like regulation, such as described for the nuclear factor of activated T-cells 1 (NFAT1) transcription factor (Salazar and Hofer, 2003), and the Sicl cyclin-dependent kinase inhibitor (Orlicky et al., 2003). Kv2.1 channel voltagedependent gating exhibits a graded response to altered phosphorylation at individual sites (Park et al., 2006), as also described for the DNA binding affinity of the Ets-1 transcription factor (Pufall et al., 2005), and Kv3.4 channel inactivation (Covarrubias et al., 1994). For such proteins, characterization of in vivo phosphorylation at individual sites is critical to understanding their regulation.

Our previous studies of recombinant Kv2.1 in heterologous cells had identified two distinct sets of phosphorylation sites, those dephosphorylated after calcineurin activation (S11, S453, 

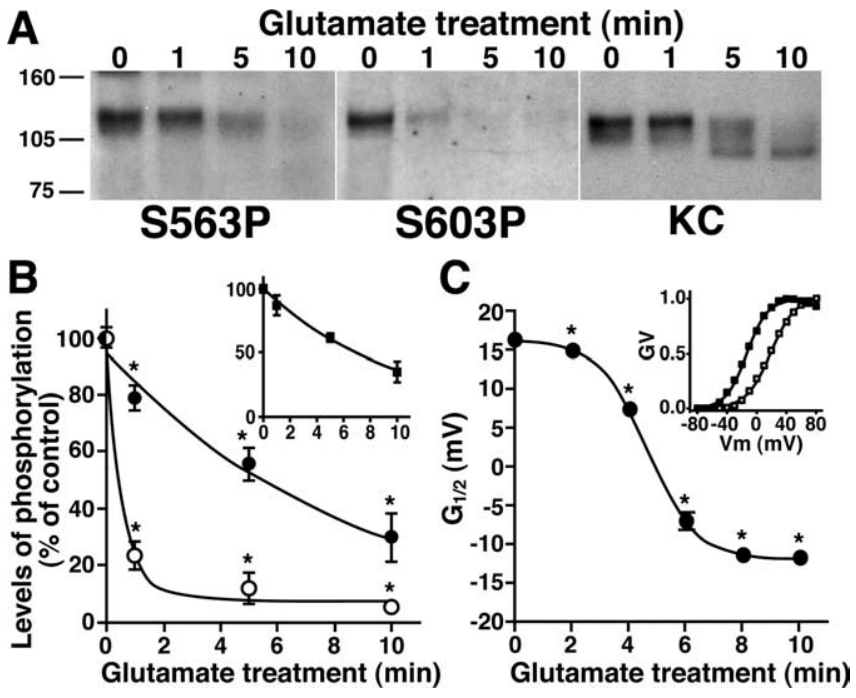

Figure 6. Temporal dissection of dephosphorylation at $\$ 563$ and $S 603$ in glutamatestimulated cultured hippocampal neurons. $A$, Cultured neurons were treated with $10 \mu \mathrm{m}$ glutamate for $0,1,5$, and $10 \mathrm{~min}$ at $37^{\circ} \mathrm{C}$. Neuronal proteins were fractionated on SDS-PAGE and resultant immunoblots were analyzed for Kv2.1 by probing with S563P, S603P, or KC as indicated. Representative blots probed with each antibody are shown. For direct comparison of phosphorylation levels, blots were stripped and reprobed with different antibodies as described in Materials and Methods. B, Time course of dephosphorylation at S563 and S603. Signals of immunoreactive bands were quantified as described in Materials and Methods, and values were converted to percentages of control values and shown as levels of phosphorylation at $\$ 563$ (filled circles) and S603 (open circles). Data are mean \pm SEM $(n=3)$. The kinetics of the glutamate-stimulated decay of Kv2.1 dephosphorylation was fitted by exponential decay curves. ${ }^{*} p<0.02$ versus control. Inset, Time course of loss of the $M_{\mathrm{r}} \approx 120 \mathrm{kDa}$ band on the $K C$ immunoblot. C, Modulation of Kv2.1-based $I_{K} \cdot G_{1 / 2}$ values (see Materials and Methods) for each time point after glutamate stimulation are shown in millivolts; values were fit with a sigmoidal curve. Inset, Representative conductance-voltage $(G-V)$ traces from control and glutamatetreated $(10 \mathrm{~min})$ neurons. $V_{\mathrm{m}}$, Membrane potential in millivolts. Data are mean $\pm \operatorname{SEM}(n=6)$. ${ }^{*} p<0.01$ versus control.

S537, S563, S603, 651, S715) and those refractory to calcineurinmediated dephosphorylation (e.g., S492, S499, S800, etc.). Here, we extend these results to show that within the set of calcineurinregulated sites are those that exhibit strikingly distinct regulation during development, and in response to changes in neuronal activity. Two of these sites (S453 and S715) are quite resistant to in vivo regulation, for example by global ischemia, whereas two others (S563, S603) are more sensitive, with the S603 site exhibiting "supersensitive" regulation. Such distinct patterns of sensitivity to in vivo regulation provide a mechanism for graded changes in Kv2.1 function and neuronal activity in response to diverse stimuli that affect cytoplasmic $\mathrm{Ca}^{2+}$ levels.

\section{Molecular mechanism underlying distinct regulation of phosphorylation at S563 and S603}

Inherent differences in these sites as substrates for calcineurin, as defined by in vitro calcineurin digestion, are the likely mechanism underlying the distinct in vivo sensitivities. Similarly, the rapid and pronounced rephosphorylation (after recovery from hyperactivity-induced dephosphorylation) and hyperphosphorylation (after suppression of neuronal activity) of S603 but not S563 appear to be mechanistically founded on differences in their relative suitability as substrates for protein kinases. These in vitro data suggest inherent differences in the structure of the phosphorylation site itself and/or the surrounding region of the Kv2.1 protein, and not more complex cellular regulation (e.g., differences between S563 and S603-phosphorylated channels in their
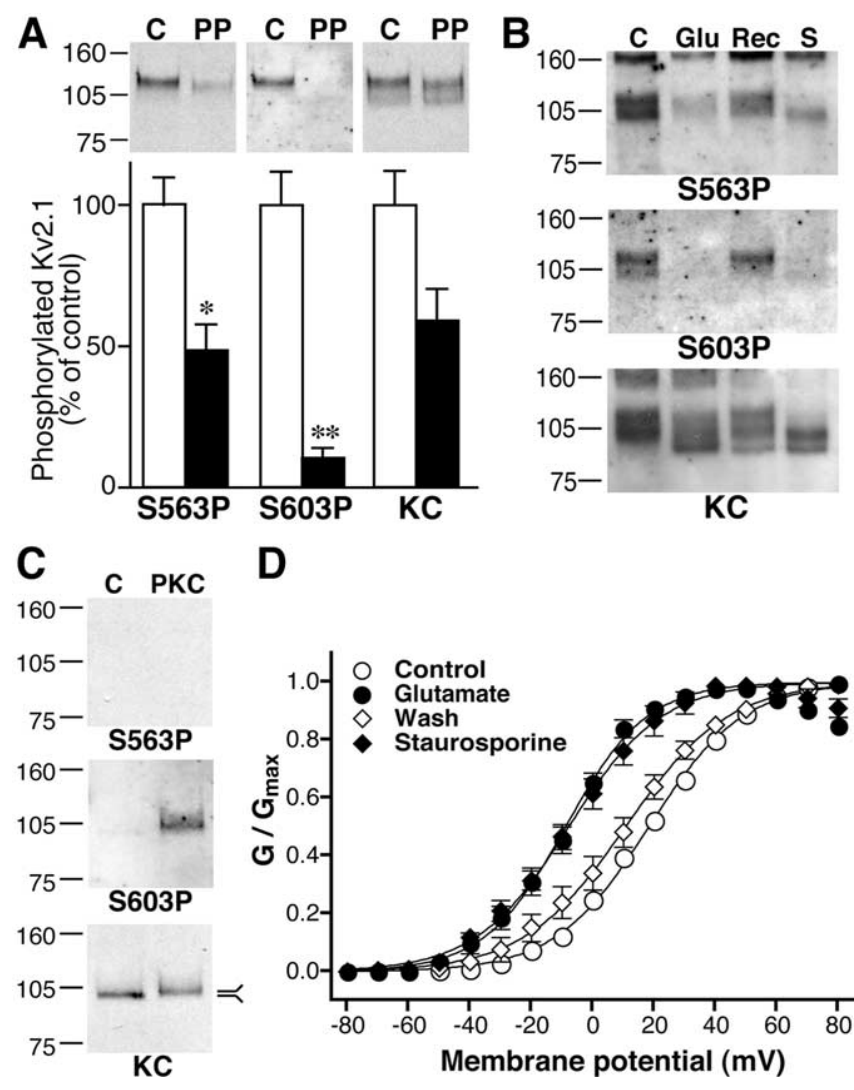

D

Figure 7. Distinct sensitivity of $S 563$ and $\mathrm{S} 603$ to calcineurin and protein kinases. $A, \mathrm{Immu}-$ nopurified Kv2.1 was incubated without (C) or with (PP) $25 \mathrm{U}$ of recombinant human calcineurin for $2 \mathrm{~h}$ at $32^{\circ} \mathrm{C}$ and analyzed on immunoblot with S563P, S603P, or KC as indicated. Bottom panel, Relative levels of Kv2.1 phosphorylation at $S 563$ and $S 603$, and of the $M_{\mathrm{r}} \approx 120 \mathrm{kDa}$ form of Kv2.1 after in vitro calcineurin dephosphorylation. Open bars, Control; filled bars, calcineurin treated. Data were from three independent experiments and are mean $\pm \operatorname{SEM}(n=3) .{ }^{*} p<$ 0.02 and ${ }^{* *} p<0.005$ versus control. $\boldsymbol{B}$, Staurosporine-mediated blockade of recovery of phosphorylation after glutamate stimulation. Cultured neurons were treated without $(C)$ or with 10 $\mu \mathrm{m}$ glutamate for $10 \mathrm{~min}$ (Glu), washed twice, and then incubated without glutamate for $2 \mathrm{~h}$ to allow recovery of phosphorylation in the absence (Rec) or presence of $100 \mathrm{~nm}$ staurosporine (S). Neuronal proteins were fractionated on SDS-PAGE, and resultant immunoblots were analyzed for Kv2.1 by immunoblotting with S563P, S603P, or KC as indicated. C, In vitro phosphorylation of Kv2.1 by purified protein kinase C. Kv2.1 was purified from RBM, dephosphorylated by digestion with AP, incubated without (C) or with purified PKC for $1 \mathrm{~h}$ at $32^{\circ} \mathrm{C}$, size fractionated by SDS-PAGE, and analyzed for Kv2.1 by immunoblotting with S563P, S603P, or KC antibodies. Lines indicate the different $M_{\mathrm{r}}$ values of Kv2.1 with or without PKC incubation. $\boldsymbol{D}$, Staurosporinemediated blockade of functional recovery of Kv2.1. Cultured neurons were treated without (open circles) or with $10 \mu \mathrm{m}$ glutamate for $10 \mathrm{~min}$ (closed circles), washed twice, and then incubated without glutamate for $2 \mathrm{~h}$ to allow recovery of phosphorylation in the absence (open diamond) or presence of staurosporine (closed diamond). Kv2.1/ $I_{\mathrm{K}}$ currents were recorded from five neurons for each condition. The membrane potential was held at $-80 \mathrm{mV}$ and depolarized from the holding potential of $-80 \mathrm{mV}$ to voltages between -70 and $+80 \mathrm{mV}$ in $10 \mathrm{mV}$ increments for $200 \mathrm{~ms}$. The plots show the conductance-voltage $(G-V)$ relationship of peak $I_{\mathrm{K}}$ currents. Data are presented as mean $\pm \operatorname{SEM}(n=5)$.

in vivo compartmentalization with calcineurin, protein kinases, and/or regulatory or inhibitory proteins) underlies the differences in in vivo regulation.

The molecular basis for the distinct preference of calcineurin for S603 over S563 is not clear. The specificity of calcineurin and other protein phosphatases for their substrates is not well understood, and their specificity is thought to be achieved mainly through cellular mechanisms detailed above (Hogan and Li, 2005). Although calcineurin binding sites have been identified on substrates such as NFAT (Li et al., 2004), they comprise a consensus sequence (PxIxIT) not present in Kv2.1. Sequences surround- 


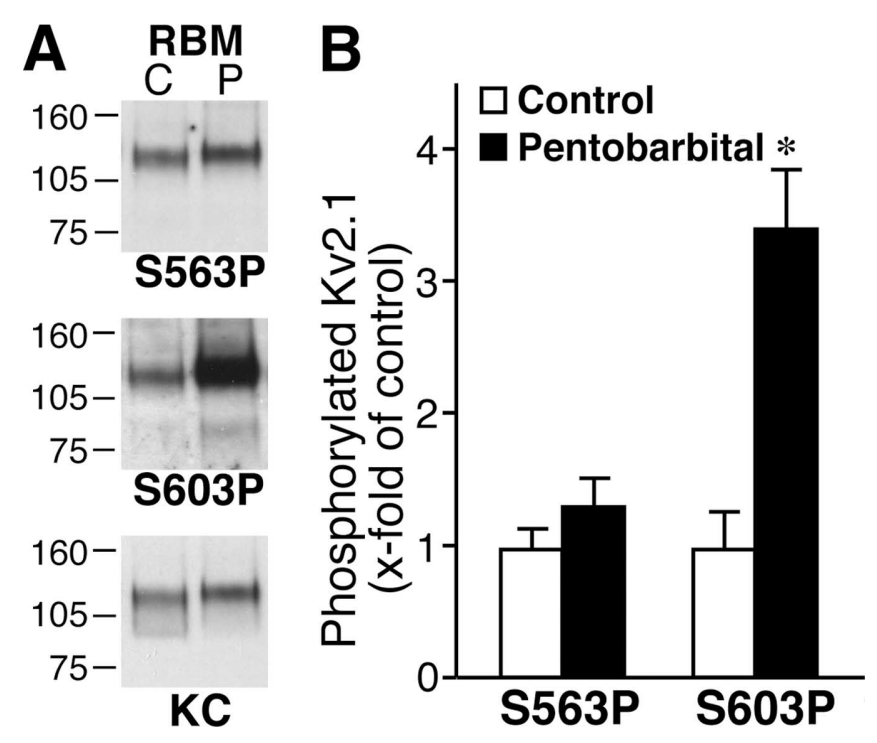

Figure 8. Hyperphosphorylation of $\mathrm{S} 603$ of $\mathrm{Kv} 2.1$ by suppressing neuronal activity in vivo. $\boldsymbol{A}$, RBM prepared from animals treated without $(C)$ or with $50 \mathrm{mg} / \mathrm{kg}$ pentobarbital $(P)$ were analyzed on immunoblot with S563P, S603P, or KC as indicated. $\boldsymbol{B}$, Levels of phosphorylation at S563 and S603 of Kv2.1 were quantified. RBM from three control (open bars) and three anesthetized animals (filled bars) were used for the analysis. Data are mean $\pm \operatorname{SEM}(n=3) .{ }^{*} p<$ 0.01 versus control.

ing the sensitive S563 (SSMPpSPVAPL) and supersensitive S603 (RFSHpSPLASL) phosphorylation sites are similar in that they are serine- and proline-rich, but sequence adjacent to more resistant sites (e.g., S715; KPVLpSPESSI) also exhibit these general characteristics. Because structural studies suggest that few features of the linear sequence are needed to specify the calcineurin site (Li et al., 2004), the molecular basis for the distinct sensitivities of the individual Kv2.1 phosphorylation sites to calcineurinmediated dephosphorylation may await structural information on the Kv2.1 C terminus.

\section{Kv2.1 phosphorylation and clustering}

Previous studies have shown that both the localization and voltage-dependent gating of Kv2.1 are regulated by activitydependent changes in phosphorylation state (Misonou et al., 2004, 2005; Mohapatra and Trimmer, 2006; Park et al., 2006). Here, we show that, in brain neurons in situ, and in cultured hippocampal neurons, clustered Kv2.1 exhibits robust S563P and S603P staining. Different brain neurons exhibit differences in the overall intensity of S563P and S603P staining, as do different clusters within a given neuron, consistent with our biochemical data that not all Kv2.1 is phosphorylated at these sites, presumably because of cell-specific differences in levels of basal activity or other factors. However, these results should be interpreted with caution because a systematic and quantitative neuroanatomical analysis of S563P and S603P staining has not been performed. Moreover, a number of the experimental interventions associated with staining procedures could potentially impact phosphorylation. Anesthetics such as pentobarbital induce Kv2.1 hyperphosphorylation, and ischemic conditions that may occur during brain isolation or perfusion induce Kv2.1 dephosphorylation, confounding analyses aimed at determining the absolute level of phosphorylation present in the living animal.

Stimuli that increase neuronal activity and/or cytoplasmic $\mathrm{Ca}^{2+}$ levels lead to dispersion of Kv2.1 clusters and loss of S563P and $\mathrm{S603P}$ staining, both in brain in vivo and in cultured neurons.
It is intriguing that S563 and S603 flank the domain (amino acids 573-598) necessary and sufficient for Kv2.1 clustering (Lim et al., 2000) and regulation of clustering by phosphorylation (Mohapatra and Trimmer, 2006). However, mutagenesis studies suggest that these sites are not in themselves determinants of clustering, because they lie outside the minimal clustering domain (Lim et al., 2000; Mohapatra and Trimmer, 2006) and S563A and S603A mutants exhibit wild-type clustering in HEK293 cells (Park et al., 2006) (K.-S. Park, D. P. Mohapatra, and J. S. Trimmer, unpublished results). The loss of S563P and S603P staining that occurs with dispersion of Kv2.1 clusters presumably reflects dephosphorylation events coincident with those regulating Kv2.1 localization.

\section{Kv2.1 phosphorylation and gating}

At early times after glutamate stimulation, when phosphorylation at S603 (but not S563) is dramatically reduced, only small (albeit significant) changes in voltage-dependent gating of neuronal $I_{\mathrm{K}}$ have occurred. Our previous studies revealed that, in heterologous cells, the activation midpoint of the S603A phosphorylation site point mutant is left-shifted by $\approx 12 \mathrm{mV}$ relative to wild-type Kv2.1, and that the effects of the mutation are through altered phosphorylation state (Park et al., 2006). The lack of a linear correlation between impact of in vivo modulation of S603 phosphorylation and the impact of S603A mutation on voltage dependence of Kv2.1 gating could be attributable to a number of plausible considerations. Because Kv2.1 comprises $\approx 80 \%$ of neuronal $I_{\mathrm{K}}$ (Murakoshi et al., 1997), with the nonKv2.1 component refractory to modulation by glutamate (Misonou et al., 2004), the functional effects of modulation of Kv2.1 at $\mathrm{S} 603$ on whole-cell $I_{\mathrm{K}}$ may be somewhat diluted by its contamination with non-Kv2.1 $I_{\mathrm{K}}$-like currents. Moreover, expression of recombinant Kv2.1 channels in heterologous cells ensures generation of a homogeneous population of homotetrameric channels. This may not be the case in neurons, where the entire neuronal Kv2.1 population does not appear to be phosphorylated at S603 under control conditions, given that suppression of activity results in $\mathrm{S} 603$ hyperphosphorylation. It is also not known how phosphorylation of individual subunits within the Kv2.1 channel tetramer affects function. For example, for calcium-activated BK channels, different phosphorylation sites can exhibit either dominant or recessive characteristics (Tian et al., 2004). These facts together may complicate attempts to linearly relate the predicted functional effects of individual phosphorylation sites as derived from studies of mutant Kv2.1 subunits expressed in heterologous cells to effects on the macroscopic Kv2.1 currents (or whole-cell outward currents) in neurons. That staurosporine effectively blocked the recovery of S603 (but not S563) phosphorylation and completely blocked the recovery of the channel function after glutamate stimulation is consistent with a potential functional role for $\mathrm{S} 603$ in neurons.

\section{S603P is a sensitive biomarker of neuronal activity}

Brain activity biomarkers are crucial to map neuronal circuits involved in specific behaviors. The phosphospecific antibody S603P represents a novel reagent for biochemical and immunohistochemical analyses of neuronal activity. Perhaps the most commonly used activity biomarkers are those that detect immediate early gene (IEG) expression (Guzowski et al., 2005). IEG expression is undetectable in resting neurons, and is rapidly (within 1-2 min) upregulated in response to increased neuronal activity. Moreover, newly synthesized IEG transcripts are rapidly cleared from the nucleus, such that restoration of resting activity levels results in not only cessation of IEG expression but also rapid ( $\approx 15 \mathrm{~min}$ ) clearing of leftover nuclear signals. However, 
one disadvantage of IEG-based biomarkers is that they are unidirectional, in that expression is undetectable in "resting" neurons that have significant levels of spontaneous basal activity.

Like IEGs, S603P immunoreactivity undergoes rapid changes in response to enhanced neuronal activity. That brief $(\approx 1 \mathrm{~min})$ exposures to ischemia in vivo, or to glutamate in culture, results in complete loss S603P immunoreactivity on neuronal Kv2.1, suggests that $\mathrm{S} 603 \mathrm{P}$ can be used in quantitative immunoblot analyses, and in double-label immunofluorescence experiments as a sensitive probe of neuronal activity. Because S603P exhibits intermediate levels of immunoreactivity against resting neurons, it can serve as a bidirectional biomarker whose signal changes in response to both elevating and depressing basal neuronal activity. The sensitive and bidirectional nature of activity-dependent changes in S603 phosphorylation suggests that important insights into neuronal activity in mammalian brain can be gained by application of the S603P antibody.

\section{References}

Antonucci DE, Lim ST, Vassanelli S, Trimmer JS (2001) Dynamic localization and clustering of dendritic Kv2.1 voltage-dependent potassium channels in developing hippocampal neurons. Neuroscience 108:69-81.

Bekkers JM (2000) Distribution and activation of voltage-gated potassium channels in cell-attached and outside-out patches from large layer 5 cortical pyramidal neurons of the rat. J Physiol (Lond) 525:611-620.

Chung HJ, Xia J, Scannevin RH, Zhang X, Huganir RL (2000) Phosphorylation of the AMPA receptor subunit GluR2 differentially regulates its interaction with PDZ domain-containing proteins. J Neurosci 20:7258-7267.

Colbert CM, Pan E (2002) Ion channel properties underlying axonal action potential initiation in pyramidal neurons. Nat Neurosci 5:533-538.

Covarrubias M, Wei A, Salkoff L, Vyas TB (1994) Elimination of rapid potassium channel inactivation by phosphorylation of the inactivation gate. Neuron 13:1403-1412.

Du J, Haak LL, Phillips-Tansey E, Russell JT, McBain CJ (2000) Frequencydependent regulation of rat hippocampal somato-dendritic excitability by the $\mathrm{K}^{+}$channel subunit Kv2.1. J Physiol (Lond) 522:19-31.

Ehlers MD (2000) Reinsertion or degradation of AMPA receptors determined by activity-dependent endocytic sorting. Neuron 28:511-525.

Guzowski JF, Timlin JA, Roysam B, McNaughton BL, Worley PF, Barnes CA (2005) Mapping behaviorally relevant neural circuits with immediateearly gene expression. Curr Opin Neurobiol 15:599-606.

Hogan PG, Li H (2005) Calcineurin. Curr Biol 15:R442-R443.

Kang J, Huguenard JR, Prince DA (2000) Voltage-gated potassium channels activated during action potentials in layer $\mathrm{V}$ neocortical pyramidal neurons. J Neurophysiol 83:70-80.

Korngreen A, Sakmann B (2000) Voltage-gated $\mathrm{K}^{+}$channels in layer 5 neocortical pyramidal neurones from young rats: subtypes and gradients. J Physiol (Lond) 525:621-639.

Levitan IB (2006) Signaling protein complexes associated with neuronal ion channels. Nat Neurosci 9:305-310.

Li H, Rao A, Hogan PG (2004) Structural delineation of the calcineurinNFAT interaction and its parallels to PP1 targeting interactions. J Mol Biol 342:1659-1674.

Lim ST, Antonucci DE, Scannevin RH, Trimmer JS (2000) A novel targeting signal for proximal clustering of the $\mathrm{Kv} 2.1 \mathrm{~K}^{+}$channel in hippocampal neurons. Neuron 25:385-397.

Maletic-Savatic M, Lenn NJ, Trimmer JS (1995) Differential spatiotemporal expression of $\mathrm{K}^{+}$channel polypeptides in rat hippocampal neurons developing in situ and in vitro. J Neurosci 15:3840-3851.

Malin SA, Nerbonne JM (2000) Elimination of the fast transient in superior cervical ganglion neurons with expression of KV4.2W362F: molecular dissection of IA. J Neurosci 20:5191-5199.

Martina M, Jonas P (1997) Functional differences in $\mathrm{Na}^{+}$channel gating between fast-spiking interneurones and principal neurones of rat hippocampus. J Physiol (Lond) 505:593-603.

Meggio F, Donella Deana A, Ruzzene M, Brunati AM, Cesaro L, Guerra B, Meyer T, Mett H, Fabbro D, Furet P, Dobrowolska G, Pinna LA (1995) Different susceptibility of protein kinases to staurosporine inhibition. Kinetic studies and molecular bases for the resistance of protein kinase CK2. Eur J Biochem 234:317-322.
Misonou H, Trimmer JS (2005) A primary culture system for biochemical analyses of neuronal proteins. J Neurosci Methods 144:165-173.

Misonou H, Mohapatra DP, Park EW, Leung V, Zhen D, Misonou K, Anderson AE, Trimmer JS (2004) Regulation of ion channel localization and phosphorylation by neuronal activity. Nat Neurosci 7:711-718.

Misonou H, Mohapatra DP, Menegola M, Trimmer JS (2005) Calcium- and metabolic state-dependent modulation of the voltage-dependent Kv2.1 channel regulates neuronal excitability in response to ischemia. J Neurosci 25:11184-11193.

Misonou H, Menegola M, Buchwalder L, Park EW, Meredith A, Rhodes KJ, Aldrich RW, Trimmer JS (2006) Immunolocalization of the $\mathrm{Ca}^{2+}$. activated $\mathrm{K}^{+}$channel Slo1 in axons and nerve terminals of mammalian brain and cultured neurons. J Comp Neurol 496:289-302.

Mohapatra DP, Trimmer JS (2006) The Kv2.1 C terminus can autonomously transfer Kv2.1-like phosphorylation-dependent localization, voltage-dependent gating, and muscarinic modulation to diverse $\mathrm{Kv}$ channels. J Neurosci 26:685-695.

Murakoshi H, Trimmer JS (1999) Identification of the Kv2.1 $\mathrm{K}^{+}$channel as a major component of the delayed rectifier $\mathrm{K}^{+}$current in rat hippocampal neurons. J Neurosci 19:1728-1735.

Murakoshi H, Shi G, Scannevin RH, Trimmer JS (1997) Phosphorylation of the $\mathrm{Kv} 2.1 \mathrm{~K}^{+}$channel alters voltage-dependent activation. Mol Pharmacol 52:821-828.

Orlicky S, Tang X, Willems A, Tyers M, Sicheri F (2003) Structural basis for phosphodependent substrate selection and orientation by the SCFCdc4 ubiquitin ligase. Cell 112:243-256.

Pal S, Hartnett KA, Nerbonne JM, Levitan ES, Aizenman E (2003) Mediation of neuronal apoptosis by Kv2.1-encoded potassium channels. J Neurosci 23:4798-4802.

Park KS, Mohapatra DP, Misonou H, Trimmer JS (2006) Graded regulation of the Kv2.1 potassium channel by variable phosphorylation. Science 313:976-979.

Pufall MA, Lee GM, Nelson ML, Kang HS, Velyvis A, Kay LE, McIntosh LP, Graves BJ (2005) Variable control of Ets-1 DNA binding by multiple phosphates in an unstructured region. Science 309:142-145.

Racine RJ (1972) Modification of seizure activity by electrical stimulation. II. Motor seizure. Electroencephalogr Clin Neurophysiol 32:281-294.

Rhodes KJ, Keilbaugh SA, Barrezueta NX, Lopez KL, Trimmer JS (1995) Association and colocalization of $\mathrm{K}^{+}$channel $\alpha$ - and $\beta$-subunit polypeptides in rat brain. J Neurosci 15:5360-5371.

Rhodes KJ, Carroll KI, Sung MA, Doliveira LC, Monaghan MM, Burke SL, Strassle BW, Buchwalder L, Menegola M, Cao J, An WF, Trimmer JS (2004) KChIPs and Kv4 $\alpha$ subunits as integral components of A-type potassium channels in mammalian brain. J Neurosci 24:7903-7915.

Salazar C, Hofer T (2003) Allosteric regulation of the transcription factor NFAT1 by multiple phosphorylation sites: a mathematical analysis. J Mol Biol 327:31-45.

Shi G, Trimmer JS (1999) Differential asparagine-linked glycosylation of voltage-gated $\mathrm{K}^{+}$channels in mammalian brain and in transfected cells. J Membr Biol 168:265-273.

Shi G, Kleinklaus AK, Marrion NV, Trimmer JS (1994) Properties of Kv2.1 $\mathrm{K}^{+}$channels expressed in transfected mammalian cells. J Biol Chem 269:23204-23211.

Sipeki S, Bander E, Ways DK, Farago A (2002) Activation of Erk1/Erk2 and transiently increased p53 levels together may account for p21 expression associated with phorbol ester-induced transient growth inhibition in HepG2 cells. Cell Signal 14:115-121.

Song P, Yang Y, Barnes-Davies M, Bhattacharjee A, Hamann M, Forsythe ID, Oliver DL, Kaczmarek LK (2005) Acoustic environment determines phosphorylation state of the Kv3.1 potassium channel in auditory neurons. Nat Neurosci 8:1335-1342.

Surmeier DJ, Foehring R (2004) A mechanism for homeostatic plasticity. Nat Neurosci 7:691-692.

Tian L, Coghill LS, McClafferty H, MacDonald SH, Antoni FA, Ruth P, Knaus HG, Shipston MJ (2004) Distinct stoichiometry of BKCa channel tetramer phosphorylation specifies channel activation and inhibition by cAMPdependent protein kinase. Proc Natl Acad Sci USA 101:11897-11902.

Trimmer JS (1991) Immunological identification and characterization of a delayed rectifier $\mathrm{K}^{+}$channel polypeptide in rat brain. Proc Natl Acad Sci USA 88:10764-10768.

Trimmer JS (1993) Expression of Kv2.1 delayed rectifier $\mathrm{K}^{+}$channel isoforms in the developing rat brain. FEBS Lett 324:205-210. 